\title{
Variations on known and recent cardinality bounds
}

\author{
F.A. Basile, M. Bonanzinga ${ }^{\dagger}$ N. Carlson ${ }^{\ddagger}$
}

\begin{abstract}
Sapirovskii [18] proved that $|X| \leq \pi \chi(X)^{c(X) \psi(X)}$, for a regular space $X$. We introduce the $\theta$-pseudocharacter of a Urysohn space $X$, denoted by $\psi_{\theta}(X)$, and prove that the previous inequality holds for Urysohn spaces replacing the bounds on celluarity $c(X) \leq \kappa$ and on pseudocharacter $\psi(X) \leq \kappa$ with a bound on Urysohn cellularity $U c(X) \leq \kappa$ (which is a weaker conditon because $U c(X) \leq c(X)$ ) and on $\theta$-pseudocharacter $\psi_{\theta}(X) \leq \kappa$ respectivly (Note that in general $\psi(\cdot) \leq \psi_{\theta}(\cdot)$ and in the class of regular spaces $\left.\psi(\cdot)=\psi_{\theta}(\cdot)\right)$. Further, in [6] the authors generalized the Dissanayake and Willard's inequality: $|X| \leq 2^{a L_{c}(X) \chi(X)}$, for Hausdorff spaces $X$ [25, in the class of $n$-Hausdorff spaces and de Groot's result: $|X| \leq 2^{h L(X)}$, for Hausdorff spaces [11, in the class of $T_{1}$ spaces (see Theorems 2.22 and 2.23 in [6]). In this paper we restate Theorem 2.22 in [6] in the class of $n$-Urysohn spaces and give a variation of Theorem 2.23 in 6] using new cardinal functions, denoted by $U W(X), \psi w_{\theta}(X)$, $\theta-a L(X), h \theta-a L(X), \theta-a L_{c}(X)$ and $\theta-a L_{\theta}(X)$. In [5] the authors introduced the Hausdorff point separating weight of a space $X$ denoted by $\operatorname{Hpsw}(X)$ and proved a Hausdorff version of Charlesworth's inequality $|X| \leq p s w(X)^{L(X) \psi(X)}$ [7. In this paper, we introduce the Urysohn point separating weight of a space $X$, denoted by $U p s w(X)$, and prove that $|X| \leq U p s w(X)^{\theta-a L_{c}(X) \psi(X)}$, for a Urysohn space $X$.
\end{abstract}

Keywords: Urysohn; $\theta$-closure; pseudocharacter; almost Lindelöf degree; Hausdorff point separating weight.

AMS Subject Classification: 54A25.

\footnotetext{
*University of Messina

${ }^{\dagger}$ University of Messina

$\ddagger$ California Lutheran University
} 


\section{Introduction}

We shall follow notations from 12 and 14 . Recall that a space $X$ is Urysohn if for every two distinct points $x, y \in X$ there are open sets $U$ and $V$ such that $x \in U, y \in V$ and $\bar{U} \cap \bar{V}=\emptyset$.

For a space $X$, we denote by $\chi(X)$ (resp., $\psi(X), \pi \chi(X), c(X), t(X)$ ) the character, (resp., pseudocharacter, $\pi$-character, celluarity, tightness) of a space $X[12$.

The $\theta$-closure of a set $A$ in a space $X$ is the set $\operatorname{cl}_{\theta}(A)=\{x \in X$ : for every neighborhood $U \ni x, \bar{U} \cap A \neq \emptyset\} ; A$ is said to be $\theta$-closed if $A=\operatorname{cl}_{\theta}(A)$ [24]. Considering the fact that the $\theta$-closure operator is not in general idempotent, Bella and Cammaroto defined in [2] the $\theta$-closed hull of a subset $A$ of a space $X$, denoted by $[A]_{\theta}$, that is the smallest $\theta$-closed subset of $X$ containing $A$. The $\theta$-tightness of $X$ at $x \in X$ is $t_{\theta}(x, X)=\min \{k$ : for every $A \subseteq X$ with $x \in \operatorname{cl}_{\theta}(A)$ there exists $B \subseteq A$ such that $|B| \leq k$ and $x \in \operatorname{cl}_{\theta}(B)$; the $\theta$-tightness of $X$ is $t_{\theta}(X)=\sup \left\{t_{\theta}(x, X): x \in X\right\}$ [8]. We have that tightness and $\theta$-tightness are independent (see Example 11 and Example 12 in [9]), but if $X$ is a regular space then $t(X)=t_{\theta}(X)$. The $\theta$-density of $X$ is $d_{\theta}(X)=\min \{k: A \subseteq X, A$ is a dense subset of $X$ and $|A| \leq k\}$. We say that a subset $A$ of $X$ is $\theta$-dense in $X$ if $\operatorname{cl}_{\theta}(A)=X$.

If $X$ is a Hausdorff space, the closed pseudocharacter of a point $x$ in $X$ is $\psi_{c}(x, X)=\min \{|\mathcal{U}|: \mathcal{U}$ is a family of open neighborhoods of $x$ and $\{x\}$ is the intersection of the closure of $\mathcal{U}$; ; the closed pseudocharacter of $X$ is $\psi_{c}(X)=\sup \left\{\psi_{c}(x, X): x \in X\right\}$ (see [19] where it is called $S \psi(X)$ ). The Urysohn pseudocharacter of $X$, denoted by $U \psi(X)$, is the smallest cardinal $k$ such that for each point $x \in X$ there is a collection $\{V(\alpha, x): \alpha<k\}$ of open neighborhoods of $x$ such that if $x \neq y$, then there exist $\alpha, \beta<k$ such that $\overline{V(\alpha, x)} \cap \overline{V(\beta, y)}=\emptyset[20$; this cardinal function is defined only for Urysohn spaces. The Urysohn-cellularity of a space $\mathrm{X}$ is $U c(X)=\sup \{|\mathcal{V}|: \mathcal{V}$ is Urysohn-cellular\} (a collection $\mathcal{V}$ of open subsets of $X$ is called Urysohncellular, if $O_{1}, O_{2}$ in $\mathcal{V}$ and $O_{1} \neq O_{2}$ implies $\overline{O_{1}} \cap \overline{O_{2}}=\emptyset$ ). Of course, $U c(X) \leq c(X)$.

The almost Lindelöf degree of a subset $Y$ of a space $X$ is $a L(Y, X)=$ $\min \{k$ : for every cover $\mathcal{V}$ of $Y$ consisting of open subsets of $X$, there exists $\mathcal{V}^{\prime} \subseteq \mathcal{V}$ such that $\left|\mathcal{V}^{\prime}\right| \leq k$ and $\left.\bigcup\left\{\bar{V}: V \in \mathcal{V}^{\prime}\right\}=Y\right\}$. The function $a L(X, X)$ is called the almost Lindelöf degree of $X$ and denoted by $a L(X)$ (see [25] and [15]). The almost Lindelof degree of $X$ with respect to closed subsets of $X$ is $a L_{c}(X)=\sup \{a L(C, X): C \subseteq X$ is closed $\}$.

For a subset $\mathrm{A}$ of a space $\mathrm{X}$ we will denote by $[A]^{\leq \lambda}$ the family of all subsets of A of cardinality $\leq \lambda$.

Sapirovskii [18] proved that $|X| \leq \pi \chi(X)^{c(X) \psi(X)}$, for a regular space $X$. 
Later Shu-Hao [19] proved that the previous inequality holds in the class of Hausdorff spaces by replacing the pseudocharacter with the closed pseudocharacter. In Section 2 we introduce the $\theta$-pseudocharacter of a Urysohn space $X$, denoted by $\psi_{\theta}(X)$ and prove the following result:

- $|X| \leq \pi \chi(X)^{U c(X) \psi_{\theta}(X)}$ for a Urysohn space $X$.

A space $X$ is $n$-Urysohn [4] (resp. n-Hausdorff [3]), $n \in \omega$, if for every $x_{1}, x_{2}, \ldots, x_{n} \in X$ there exist open subsets $U_{1}, U_{2}, \ldots, U_{n}$ of $X$ such that $x_{1} \in U_{1}, x_{2} \in U_{2}, \ldots, x_{n} \in U_{n}$ and $\bigcap_{i=1}^{n} \overline{U_{i}}=\emptyset\left(\operatorname{resp}, \bigcap_{i=1}^{n} U_{i}=\emptyset\right)$. In [6] the authors generalized the Dissanayake and Willard's inequality: $|X| \leq 2^{a L_{c}(X) \chi(X)}$, for Hausdorff spaces $X[25$, in the class of $n$-Hausdorff spaces and de Groot's result: $|X| \leq 2^{h L(X)}$, for Hausdorff spaces [11, in the class of $T_{1}$ spaces. In particular, they used two new cardinal functions, denoted by $H W(X), \psi w(X)$, to obtain the following results:

- If $X$ is a $T_{1} n$-Hausdorff $(n \in \omega)$ space, then $|X| \leq H W(X) 2^{a L_{c}(X) \chi(X)}$.

- If $X$ is a $T_{1}$ space, then $|X| \leq H W(X) \psi w(X)^{h a L(X)}$.

In Section 3 we introduce new cardinal functions, denoted by $U W(X), \psi w_{\theta}(X)$, $\theta-a L(X), h \theta-a L(X), \theta-a L_{c}(X)$ and $\theta-a L_{\theta}(X)$ such that $H W(X) \leq U W(X)$, $\psi w(X) \leq \psi w_{\theta}(X)$ and $\theta-a L(X) \leq a L(X)$, restate Theorem 2.22 in [6] in the class of $n$-Urysohn spaces and give a variation of Theorem 2.23 in [6]. In particular, we prove the following results:

- If $X$ is a $T_{1} n$-Urysohn $(n \in \omega)$ space, then $|X| \leq U W(X) 2^{\theta-a L_{\theta}(X) \chi(X)}$.

- If $X$ is a $T_{1}$ space then $|X| \leq U W(X) \psi w_{\theta}(X)^{h \theta-a L(X)}$.

In [5] the authors introduced the Hausdorff point separating weight of a space $X$ denoted by $\operatorname{Hpsw}(X)$ and proved a Hausdorff version of Charlesworth's inequality $|X| \leq p s w(X)^{L(X) \psi(X)}$ [7]. In a similar way, in Section 4 we introduce Urysohn point separating weight of a space $X$, denoted by $H p s w(X)$, and prove the following result:

- If $X$ is a Urysohn space, then $|X| \leq U p s w(X)^{\theta-a L_{c}(X) \psi(X)}$.

\section{A generalization of Sapirovskii's inequality $|X| \leq \pi \chi(X)^{c(X) \psi(X)}$.}

Definition 2.1. If $X$ is a Urysohn space, we define $\theta$-pseudocharacter of a point $x \in X$ the smallest cardinal $k$ such that $\{x\}$ is the intersection of the $\theta$ closure of the closure of a family of open neighborhood of $x$ having cardinality less or equal to $k$; we denote it with $\psi_{\theta}(x, X)$. The $\theta$-pseudocharacter of $X$ is:

$$
\psi_{\theta}(X)=\sup \left\{\psi_{\theta}(x, X): x \in X\right\} .
$$

The following result is trivial: 
Proposition 2.1. $X$ is a Urysohn space iff for every $x \in X,\{x\}$ is the intersection of the $\theta$-closure of the closure of a family of open neighborood of $x$.

Proof. Let $X$ be a Urysohn space and $x \in X$. For every $y \in X \backslash\{x\}$, there exist $U_{y}$ and $V_{y}$ open disjoint subsets of $X$ such that $x \in U_{y}, y \in V_{y}$ and $\overline{U_{y}} \cap \overline{V_{y}}=\emptyset$. So, y $\notin c l_{\theta}\left(\overline{U_{y}}\right)$ and $\{x\}=\bigcap_{y \in X \backslash\{x\}} c l_{\theta}\left(\overline{U_{y}}\right)$. Viceversa let $x, y$ be distinct points of $X$. By hypothesis there exists an open neighbourhood $V$ of $x$ such that $y \notin c l_{\theta}(\bar{V})$. Then there exists an open subset $U$ of $X$ such that $y \in U$ and $\bar{U} \cap \bar{V}=\emptyset$. So $X$ is Urysohn.

We have that:

$$
\psi(X) \leq \psi_{c}(X) \leq \psi_{\theta}(X) \leq U \psi(X) \leq \chi(X) .
$$

Since for a regular space $X, c_{\theta}(A)=\bar{A}$ for every $A \subseteq X$ [13], we have that for a regular space $X, \psi_{c}(X)=\psi_{\theta}(X)$. In general this need not be true for non regular spaces. Indeed if we consider $\mathbb{R}$ with the countable complement topology we have that $\overline{\mathbb{Q}} \neq \operatorname{cl}_{\theta}(\mathbb{Q})$.

Question 2.1. Is there a Urysohn space such that $\psi_{c}(X)<\psi_{\theta}(X)$ ?

It was proved in [2] that for Urysohn spaces, $\left|c l_{\theta}(A)\right| \leq|A|^{\chi(X)}$ for every $A \subseteq X$ and further this inequality was used for the estimation of cardinality of Lindelöf spaces. Since $t_{\theta}(X) \psi_{\theta}(X) \leq \chi(X)$, the following proposition improves the result in [2]. (Note that if $X=\omega \cup\{p\}$, with $p \in \omega^{*}$, we have that $\aleph_{0}=t_{\theta}(X) \psi_{\theta}(X)<\chi(X)$.)

Proposition 2.2. Let $X$ be a Urysohn space such that $t_{\theta}(X) \psi_{\theta}(X) \leq k$. Then for every $A \subseteq X$ we have that $\left|\operatorname{cl}_{\theta}(A)\right| \leq|A|^{k}$.

Proof. Let $x \in c l_{\theta}(A)$, since $\psi_{\theta}(X) \leq k$ there exist a family $\left\{U_{\alpha}(x)\right\}_{\alpha<k}$ of neighborhood of $x$ such that $\{x\}=\bigcap_{\alpha<k} c l_{\theta}\left(\overline{U_{\alpha}(x)}\right)$. We want to prove that $x \in c l_{\theta}\left(\overline{U_{\alpha}(x)} \cap A\right), \forall \alpha<k$. Let $U$ be a neighborhood of $x$ and $\alpha<k$. Then $\emptyset \neq \overline{U \cap U_{\alpha}(x)} \cap A \subseteq \bar{U} \cap \overline{U_{\alpha}(x)} \cap A$. This shows that $x \in \operatorname{cl}_{\theta}\left(\overline{U_{\alpha}(x)} \cap A\right)$. Since $t_{\theta}(X) \leq k$, there exists $A_{\alpha} \subset \overline{U_{\alpha}(x)} \cap A$ such that $\left|A_{\alpha}\right| \leq k$ and $x \in c l_{\theta}\left(A_{\alpha}\right)$. Then $\{x\}=\bigcap_{\alpha<k} c l_{\theta}\left(A_{\alpha}\right)$ and $\left\{A_{\alpha}\right\}_{\alpha<k} \in$ $[[A] \leq k] \leq k$, so $\left|c l_{\theta}(A)\right| \leq\left|\left[[A]^{\leq k}\right] \leq k\right|=|A|^{k}$.

Corollary 2.1. [2] If $X$ is a Urysohn space then for every $A \subseteq X$ we have that $\left|c l_{\theta}(A)\right| \leq|A|^{\chi(X)}$.

The following result is the analogue of 2.20 in [16] in the case of Urysohn spaces.

Corollary 2.2. If $X$ is a Urysohn space then $|X| \leq d_{\theta}(X)^{t_{\theta}(X) \psi_{\theta}(X)}$. 
Proof. If $A$ is $\theta$-dense subset of $X$, i.e. $\operatorname{cl}_{\theta}(A)=X$, we have that $|A| \leq d_{\theta}(X)$ and from the above theorem we have that $\left|c l_{\theta}(A)\right| \leq|A|^{t_{\theta}(X) \psi_{\theta}(X)}$, so $|X| \leq$ $d_{\theta}(X)^{t_{\theta}(X) \psi_{\theta}(X)}$.

The authors know that I. Gotchev obtained independently the results given in Proposition 2.2 and Corollary 2.2 .

Now we prove the following result:

Lemma 2.1. Let $X$ be a topological space, $\mathcal{B}$ a $\pi$-base for $X$ and $\mathcal{W}$ a family of open sets. Let $\mathcal{M}$ be a maximal Urysohn cellular subfamily of $\{U \in \mathcal{B}: U \subseteq W$ for some $W \in \mathcal{W}\}$. Then $\operatorname{cl}_{\theta}(\bigcup \overline{\mathcal{M}}) \supseteq \bigcup \mathcal{W}$.

Proof. Using Zorn's Lemma we can say that there exists a maximal Urysohncellular subfamily $\mathcal{M}$ of $\{U \in \mathcal{B}: U \subseteq W$ for some $W \in \mathcal{W}\}$. We want to prove that $\operatorname{cl}_{\theta}(\bigcup \overline{\mathcal{M}}) \supseteq \bigcup \mathcal{W}$. Assume, by the way of contradiction, that $c_{\theta}(\bigcup \overline{\mathcal{M}}) \not \supset \bigcup \mathcal{W}$. Let $x \in \bigcup \mathcal{W}$ such that $x \notin c l_{\theta}(\bigcup \overline{\mathcal{M}})$. Then there exists an open set $U$ such that $x \in U$ such that $\bar{U} \cap \bar{M}=\emptyset, \forall M \in \mathcal{M}$. So $x \notin M, \forall M \in \mathcal{M}$. Let $W \in \mathcal{W}$ such that $x \in W . \mathcal{M} \cup\{U \cap W\}$ is a Urysohn cellular family. Since $\mathcal{B}$ is a $\pi$-base for $X$ and $U \cap W$ is an open set containing $x$, there exists $B \in \mathcal{B}$ such that $B \subseteq U \cap W$, so $\mathcal{M}^{\prime}=\mathcal{M} \cup\{B\}$ is a Urysohn cellular subfamily of $\{U \in \mathcal{B}: U \subseteq W$ for some $W \in \mathcal{W}\}$ containing $\mathcal{M}$; a contradiction.

Theorem 2.1. Let $X$ be a Urysohn space. Then $|X| \leq \pi \chi(X)^{U c(X) \psi_{\theta}(X)}$.

Proof. Let $\pi \chi(X)=\lambda$ and $U c(X) \psi_{\theta}(X)=k$; for each $p \in X$, let $\mathcal{U}_{p}$ be a local $\pi$-base at $p$ such that $\left|\mathcal{U}_{p}\right| \leq \lambda$.

Construct an increasing chain $\left\{A_{\alpha}: \alpha<k^{+}\right\}$of subsets of $X$ and a sequence $\left\{\mathcal{U}_{\alpha}: 0<\alpha<k^{+}\right\}$of open collections in $X$ such that:

1. $\left|A_{\alpha}\right| \leq \lambda^{k}, 0 \leq \alpha<k^{+}$;

2. $\mathcal{U}_{\alpha}=\left\{V \in \mathcal{U}_{p}: p \in \bigcup_{\beta<\alpha} A_{\beta}\right\}, 0<\alpha<k^{+}$;

3. for each $\gamma<k$, if $\mathcal{V}_{\gamma} \in\left[\mathcal{U}_{\alpha}\right]^{\leq k}$ and $W=\bigcup_{\gamma<k} c l_{\theta}\left(\bigcup \overline{\mathcal{V}_{\gamma}}\right) \neq X$, then $A_{\alpha} \backslash W \neq \emptyset$.

The construction is by trasfinite induction. Let $0<\alpha<k^{+}$and assume that $\left\{A_{\beta}: \beta<\alpha\right\}$ has already been constructed. Then $\mathcal{U}_{\alpha}$ is defined by 2 ., i.e., we put $\mathcal{U}_{\alpha}=\left\{V: \exists p \in \bigcup_{\beta<\alpha} A_{\beta}, V \in \mathcal{U}_{p}\right\}$. It follows that $\left|\mathcal{U}_{\alpha}\right| \leq \lambda^{k}$. If $\left\{\mathcal{V}_{\gamma}\right\}_{\gamma<k} \in\left[\left[\mathcal{U}_{\alpha}\right]^{\leq k}\right]^{\leq k}$ and $W=\bigcup_{\gamma<k} c l_{\theta}\left(\bigcup \overline{\mathcal{V}_{\gamma}}\right) \neq X$, then we can choose one point of $X \backslash W$. Let $S_{\alpha}$ be the set of points chosen in this way. Note that $\left|\left[\left[\mathcal{U}_{\alpha}\right] \leq k\right] \leq k\right| \leq \lambda^{k}$. Define $A_{\alpha}$ to be the set $S_{\alpha} \cup\left(\bigcup_{\beta<\alpha} A_{\beta}\right)$. Then $A_{\alpha}$ satisfies 1., and 3. is also satisfied if $\beta \leq \alpha$. This completes the construction. 
Now let $S=\bigcup_{\alpha<k^{+}} A_{\alpha}$; then $|S| \leq k^{+} \lambda^{k}=\lambda^{k}$. The proof is complete if $S=X$. Suppose not and let $p \in X \backslash S$; since $\psi_{\theta}(X) \leq k$, there exist open neighbourhoods $\left\{U_{\alpha}\right\}_{\alpha<k}$ of $p$ such that $\{p\}=\bigcap_{\alpha<k} c l_{\theta}\left(\overline{U_{\alpha}}\right)$. For each $\alpha<k$, let $V_{\alpha}=X \backslash c l_{\theta}\left(\overline{U_{\alpha}}\right)$. Then $S=\bigcup_{\alpha<k} V_{\alpha} \cap S$. Fix $\alpha<k$. For each $q \in V_{\alpha} \cap S$, there exists $V_{q} \in \mathcal{U}_{q}$ such that $\overline{V_{q}} \cap \overline{U_{\alpha}}=\emptyset$ (from the definition of $V_{\alpha}$ ). We have that $\left\{V \in \mathcal{U}_{q}: V \subseteq V_{q}\right\}$ is a local $\pi$-base at $q$. Since $q \in \overline{\bigcup\left\{V \in \mathcal{U}_{q}: V \subseteq V_{q}\right\}}$, we have that $S \cap V_{\alpha} \subseteq$ $\bigcup_{q \in S \cap V_{\alpha}} \overline{\bigcup\left\{V \in \mathcal{U}_{q}: V \subseteq V_{q}\right\}} \subseteq \overline{\bigcup\left\{V: V \in \mathcal{U}_{q}, V \subseteq V_{q}, q \in S \cap V_{\alpha}\right\}}$. We put $\mathcal{W}_{\alpha}=\left\{V: V \in \mathcal{U}_{q}, V \subseteq V_{q}, q \in S \cap V_{\alpha}\right\}$. Since $U c(X) \leq k$, by Lemma 2.1 we have that $\forall \alpha<k$ there exists a maximal Urysohn cellular family $\mathcal{W}_{\alpha}^{\prime} \in\left[\mathcal{W}_{\alpha}\right]^{\leq k}$ such that $c l_{\theta}\left(\bigcup \overline{\mathcal{W}_{\alpha}^{\prime}}\right) \supseteq \bigcup \mathcal{W}_{\alpha}$. Since $c l_{\theta}\left(\bigcup \overline{\mathcal{W}_{\alpha}^{\prime}}\right)$ is closed, it follows that $S \cap V_{\alpha} \subseteq \overline{\cup \mathcal{W}_{\alpha}} \subseteq \operatorname{cl}_{\theta}\left(\bigcup \mathcal{W}_{\alpha}^{\prime}\right) \subseteq c_{\theta}\left(\bigcup_{q \in S \cap V_{q}} \overline{V_{q}}\right)$. Then, since $\left(\bigcup_{q \in S \cap V_{\alpha}} \overline{V_{q}}\right) \cap \overline{U_{\alpha}}=\emptyset$ and $p \notin c l_{\theta}\left(\bigcup_{q \in S \cap V_{\alpha}} \overline{V_{q}}\right)$, we have that $p \notin c l_{\theta}\left(\bigcup \overline{\mathcal{W}_{\alpha}^{\prime}}\right)$. Put $W=\bigcup_{\alpha<k} c l_{\theta}\left(\bigcup \overline{\mathcal{W}_{\alpha}^{\prime}}\right)$. Since $\mid\left\{V: V \in \mathcal{W}_{\alpha}^{\prime}\right.$ for some $\alpha<k\} \mid \leq k k=k<k^{+}$, there is an $\alpha_{0}<k^{+}$such that $\mathcal{W}_{\alpha}^{\prime} \in\left[\mathcal{U}_{\alpha_{0}}\right] \leq k$ for each $\alpha<k$. Hence, by 3., one has $A_{\alpha_{0}} \backslash W \neq \emptyset$. But $W \supseteq \bigcup_{\alpha<k}\left(V_{\alpha} \cap S\right)=S$ and $A_{\alpha_{0}} \backslash W \subseteq S \backslash W=\emptyset$; a contradiction.

Corollary 2.3. [18] Let $X$ be a regular space. Then $|X| \leq \pi \chi(X)^{c(X) \psi(X)}$.

\section{Variations of the Dissanayake and Willard's inequality $|X| \leq 2^{a L_{c}(X) \chi(X)}$ and of the de Groot's inequality $|X| \leq 2^{h L(X)}$ in the class of $T_{1}$ spaces.}

In Proposition 2.1 it was shown that Urysohn axiom is equivalent to $\{x\}=$ $\bigcap\left\{\operatorname{cl}_{\theta}(\bar{U}): U\right.$ open, $\left.x \in U\right\}$, for every point $x$ of the space. The following example shows that in spaces which are not Urysohn the previous intersection can be large.

Example 3.1. Any infinite space $X$ with the cofinite topology is a $T_{1}$, not Hausdorff space for which there is a point $x$ such that $\bigcap\left\{c_{\theta}(\bar{U}): x \in U\right\}$ has large cardinality.

The example above gives a motivation to introduce the following definition:

Definition 3.1. Let $X$ be a $T_{1}$ topological space and for all $x \in X$, let

$$
U w(x)=\bigcap\left\{c l_{\theta}(\bar{U}): x \in U, U \text { open }\right\} .
$$


The Urysohn width is:

$$
U W(X)=\sup \{|U w(x)|: x \in X\} .
$$

It is clear that if $X$ is a Urysohn space then $U W(X)=1$.

Recall that $H W(X)=\sup \{|H w(x)|: x \in X\}$ is the Hausdorff width, where $H w(x)=\bigcap\{\bar{U}: x \in U, U$ open $\}$ [6]. Since the $\theta$-closure of a set contains its closure we have that $H W(X) \leq U W(X)$.

Question 3.1. Is $H W(X)=U W(X)$ in some class of non regular spaces?

Definition 3.2. [6] Let $X$ be a space and $x \in X$.

$$
\begin{gathered}
\psi w(x)=\min \left\{\left|\mathcal{U}_{x}\right|: \bigcap\left\{\bar{U}: U \in \mathcal{U}_{x}\right\}=H w(x), \mathcal{U}_{x}\right. \text { is a } \\
\text { family of open neighborhood of } x\} ;
\end{gathered}
$$

and

$$
\psi w(X)=\sup \{\psi w(x): x \in X\} .
$$

Similarly, we introduce the following definition.

Definition 3.3. Let $X$ be a space and $x \in X$.

$$
\begin{gathered}
\psi w_{\theta}(x)=\min \left\{\left|\mathcal{U}_{x}\right|: \bigcap\left\{c_{\theta}(\bar{U}): U \in \mathcal{U}_{x}\right\}=U w(x), \mathcal{U}_{x}\right. \text { is a } \\
\text { family of open neighborhood of } x\} ;
\end{gathered}
$$

and

$$
\psi w_{\theta}(X)=\sup \left\{\psi w_{\theta}(x): x \in X\right\} .
$$

Of course, if $X$ is a $T_{1}$ space then $\psi w(X) \leq \psi w_{\theta}(X) \leq \chi(X)$; further if $X$ is a Urysohn space then we have that $\psi w_{\theta}(X)=\psi_{\theta}(X)$.

We introduce the following definition:

Definition 3.4. Let $Y$ be a subset of a space $X$.

The $\theta$-almost Lindelöf degree of a subset $Y$ of a space $X$ is

$\theta-a L(Y, X)=\min \{k$ : for every cover $\mathcal{V}$ of $Y$ consisting of open subsets of $X$, there exists $\mathcal{V}^{\prime} \subseteq \mathcal{V}$ such that $\left|\mathcal{V}^{\prime}\right| \leq k$ and $\left.\bigcup\left\{c l_{\theta}(\bar{V}): V \in \mathcal{V}^{\prime}\right\}=Y\right\}$.

The function $\theta-a L(X, X)$ is called $\theta$-almost Lindelöf degree of the space $X$ and denoted by $\theta-a L(X)$.

The $\theta$-almost Lindelöf degree with respect to closed subsets of $X$, denoted by $\theta-a L_{c}(X)$, is the cardinal $\sup \{\theta-a L(C, X): C \subseteq X$ is closed $\}$.

The $\theta$-almost Lindelöf degree with respect to $\theta$-closed subsets of $X$, denoted by $\theta-a L_{\theta}(X)$, is the cardinal $\sup \{\theta-a L(B, X): B \subseteq X$ is $\theta$-closed $\}$. 
Of course $\theta$ - $a L(X) \leq a L(X)$, for every space $X$. Using a slight modification of Example 2.3 in [1] we prove that the previous inequality can be strict.

Example 3.2. A space $X$ such that $\theta-a L(X)<a L(X)$.

Let $k$ be any uncountable cardinal, let $\mathbb{Q}$ be the set of all the rationals and let $\mathbb{P}$ be the set of the irrationals. Put $X=(\mathbb{Q} \times k) \cup \mathbb{P}$. We topologized $X$ as follows. If $q \in \mathbb{Q}$ and $\alpha<k$ then a neighborhood base at $(q, \alpha)$ is $\mathcal{U}(q, \alpha)=\left\{U_{n}(q, \alpha): n \in \omega\right\}$ where

$$
U_{n}(q, \alpha)=\left\{(r, \alpha): r \in \mathbb{Q} \text { and }|r-q|<\frac{1}{n}\right\} .
$$

If $p \in \mathbb{P}$ a neighborhood base at $p$ takes the form:

$$
\left\{\left\{b \in \mathbb{P}:|b-p|<\frac{1}{n}\right\} \cup\left\{(q, \alpha): \alpha<k \text { and }|q-p|<\frac{1}{n}\right\}: n \in \omega\right\} .
$$

For every $q \in \mathbb{Q}, \alpha<k$ and $n \in \omega$ we have that:

$$
\overline{U_{n}(q, \alpha)}=U_{n}(q, \alpha) \bigcup\left\{(r, \alpha): r \in \mathbb{Q},|r-q|<\frac{1}{n}\right\} \bigcup\left\{p \in \mathbb{P}:|q-p|<\frac{1}{n}\right\} ;
$$

and:

$$
c l_{\theta}\left(\overline{U_{n}(q, \alpha)}\right)=\overline{U_{n}(q, \alpha)} \bigcup\left\{(r, \beta):|r-q|<\frac{1}{n}, \beta<k \text { and } \beta \neq \alpha\right\} .
$$

Let $\alpha<k$, we have that $X=\bigcup_{q \in \mathbb{Q}} c l_{\theta}(\overline{\mathcal{U}(q, \alpha)})$ and so $\theta-a L(X)=\aleph_{0}$ but we have that $a L(X)=2^{\aleph_{0}}$.

It is easy to show that the almost Lindelöf degree is hereditary with respect to $\theta$-closed subsets. It is natural to ask:

Question 3.2. Is the $\theta$-almost Lindelöf degree hereditary with respect to $\theta$-closed subsets?

We find out (Proposition 3.1) that the $\theta$-almost Lindelöf degree is hereditary with respect to a new class of spaces that we call $\gamma$-closed.

Definition 3.5. Let $X$ be a topological space and $A \subseteq X$. The $\gamma$-closure of the set $A$ is

$c l_{\gamma}(A)=\left\{x:\right.$ for every open neighborhood of $\left.X, c_{\theta}(\bar{U}) \cap A \neq \emptyset\right\}$. $A$ is said to be $\gamma$-closed if $A=\operatorname{cl}_{\gamma}(A)$.

The following example shows that the $\gamma$-closure and the $\theta$-closure of a subset of a topological space can be different. 
Example 3.3. A Urysohn space $X$ having a subset $Y$ such that $c l_{\gamma}(Y) \neq$ $c l_{\theta}(Y)$.

Proof. Let $\mathbb{R}=A \cup B \cup C \cup D$ where $A, B, C, D$ are pairwise disjoint and each is dense in $\mathbb{R}$. Let $A^{\prime}$ be a topological copy of $A$; points in $A^{\prime}$ are denoted as $a^{\prime}$ where $a \in A$.

Let $a, b \in \mathbb{R}$. A base for $X$ is generated by these families of open sets:

(1) $\{(a, b) \cap A: a, b \in \mathbb{R}, a<b\}$

(2) $\{(a, b) \cap C: a, b \in \mathbb{R}, a<b\}$,

(3) $\left\{(a, b) \cap A^{\prime}: a, b \in \mathbb{R}, a<b\right\}$,

(4) $\{(a, b) \cap(A \cup B \cup C): a, b \in \mathbb{R}, a<b\}$, and

(5) $\left\{(a, b) \cap\left(C \cup D \cup A^{\prime}\right): a, b \in \mathbb{R}, a<b\right\}$.

Note that for every $a, b \in \mathbb{R}, \overline{(a, b) \cap A}=[a, b] \cap(A \cup B), \overline{(a, b) \cap A^{\prime}}=$ $[a, b] \cap\left(A^{\prime} \cup D\right), \overline{(a, b) \cap C}=[a, b] \cap(B \cup C \cup D), c l_{\theta}(\overline{(a, b) \cap A)}=[a, b] \cap$ $(A \cup B \cup C)$ and $c l_{\theta}\left(\overline{(a, b) \cap A^{\prime}}\right)=[a, b] \cap\left(A^{\prime} \cup D \cup C\right)$. For these reasons we can say that if $a, b \in \mathbb{R}$ and if we put $Y=(a, b) \cap C$, we have that $c l_{\theta}(Y)=[a, b] \cap(B \cup C \cup D)$ and $\operatorname{cl}_{\gamma}(Y)=[a, b] \cap\left(A \cup B \cup C \cup D \cup A^{\prime}\right)$.

We have the following:

Proposition 3.1. The $\theta$-almost Lindelöf degree is hereditary with respect to $\gamma$-closed subsets.

Proof. Let $X$ be a topological space such that $\theta-a L(X) \leq k$ and let $C \subseteq X$ be $\gamma$-closed set. $\forall x \in X \backslash C$ we have that there exists an open neighborhood $U_{x}$ of $x$ such that $\operatorname{cl}_{\theta}(\bar{U}) \subseteq X \backslash C$. Let $\mathcal{U}$ be a cover of $C$ consisting of open subsets of $X$. Then $\mathcal{V}=\mathcal{U} \cup\left\{U_{x}: x \in X \backslash C\right\}$ is an open cover of $X$ and since $\theta-a L(X) \leq k$, there exists $\mathcal{V}^{\prime} \in[\mathcal{V}]^{\leq k}$ such that $X=\bigcup\left\{c l_{\theta}(\bar{V}): V \in \mathcal{V}^{\prime}\right\}$. Then there exists $\mathcal{V}^{\prime \prime} \in[\mathcal{U}]^{\leq k}$ such that $C \subseteq \bigcup\left\{c l_{\theta}(\bar{V}): V \in \mathcal{V}^{\prime \prime}\right\}$; this proves that $\theta-a L(C) \leq k$.

Now we use $U W(X)$ and $\theta-a L_{\theta}(X)$ to restate Theorem 2.22 in [6] in the class of $n$-Uysohn spaces. The proof follows step by step the proof of Theorem 2.22 in [6].

Theorem 3.1. If $X$ is a $T_{1} n$-Urysohn $(n \in \omega)$ space, then $|X| \leq U W(X) 2^{\theta-a L_{\theta}(X) \chi(X)}$.

Proof. Let $U W(X) \leq k, \theta-a L_{\theta}(X) \chi(X) \leq \tau$. For all $x \in X$, let $\mathcal{U}_{x}$ be a local base and $\left|\mathcal{U}_{x}\right| \leq \tau$. Note that for all $x \in X, U w(x)=\bigcap\left\{c l_{\theta}(\bar{U}): U \in \mathcal{U}_{x}\right\}$. Construct $\left\{H_{\alpha}: \alpha \in \tau^{+}\right\}$and $\left\{\mathcal{B}_{\alpha}: \alpha \in \tau^{+}\right\}$such that:

1. $H_{\alpha} \subset H_{\beta} \subset X$, for all $\alpha, \beta \in \tau^{+}$;

2. $H_{\alpha}$ is $\theta$-closed for all $\alpha \in \tau^{+}$; 
3. $\left|H_{\alpha}\right| \leq 2^{\tau}$ for all $\alpha \in \tau^{+}$;

4. if $\left\{H_{\beta}: \beta \in \alpha\right\}$ are defined for some $\alpha \in \tau^{+}$, then $\mathcal{B}_{\alpha}=\bigcup\left\{\mathcal{U}_{x}: x \in\right.$ $\left.\bigcup\left\{H_{\beta}: \beta \in \alpha\right\}\right\}$

5. if $\alpha \in \tau^{+}$and $\mathcal{W} \in\left[\mathcal{B}_{\alpha}\right]^{\leq \tau}$ is such that $X \backslash\left(\bigcup\left\{c l_{\theta}(\bar{U}): U \in \mathcal{W}\right\}\right) \neq \emptyset$ then $H_{\alpha} \backslash\left(\bigcup\left\{c l_{\theta}(\bar{U}): U \in \mathcal{W}\right\}\right) \neq \emptyset$.

Let $\alpha \in \tau^{+}$and $\left\{H_{\beta}: \beta \in \alpha\right\}$ be already defined. For all $\mathcal{W}$ as in 5., choose a point $x(\mathcal{W}) \in X \backslash\left(\bigcup\left\{c l_{\theta}(\bar{U}): U \in \mathcal{W}\right\}\right)$ and let $C_{\alpha}$ be the set of these points. Let $H_{\alpha}=\left[\bigcup\left\{H_{\beta}: \beta \in \alpha\right\} \cup C_{\alpha}\right]_{\theta}$. Considering the fact that if $X$ is a $n$-Urysohn space we have that for every $A \subseteq X,\left|[A]_{\theta}\right| \leq|A|^{\chi(X)}$ [4] we have that $\left|H_{\alpha}\right| \leq 2^{\tau}$. Let $H=\bigcup\left\{H_{\beta}: \beta \in \tau^{+}\right\}$. Since $t_{\theta}(X) \leq \chi(X) \leq \tau$, $\tau^{+}$is regular and $\left\{H_{\alpha}: \alpha \in \tau^{+}\right\}$is an increasing family of my $\theta$-closed sets of lenght $\tau^{+}$, we have that $H$ is $\theta$-closed. Also $|H| \leq 2^{\tau}$. Let $H^{*}=\bigcup\{U w(x)$ : $x \in H\} \supseteq H$. Then $\left|H^{*}\right| \leq k 2^{\tau}$.

We want to prove that $X=H^{*}$. Suppose that there exists a point $q \in X \backslash H^{*} \subset X \backslash H$. Then for all $x \in H$ there is $U(x) \in \mathcal{U}_{x}$ such that $q \notin c l_{\theta}(\overline{U(x)})$. From $\theta-a L_{\theta}(X) \leq \tau$ choose $H^{\prime} \in[H]^{\leq \tau}$ such that $H \subseteq$ $\bigcup\left\{c l_{\theta}(\overline{U(x)}): \quad x \in H^{\prime}\right\}$. Then $H^{\prime} \subseteq H_{\alpha}$ for some $\alpha \in \tau^{+}$and hence $\mathcal{W}=\left\{c l_{\theta}(\overline{U(x)}): x \in H^{\prime}\right\} \in\left[\mathcal{B}_{\alpha+1}\right]^{\leq \tau}$ and $q \in X \backslash\left(\bigcup\left\{c l_{\theta}(\bar{U}): U \in \mathcal{W}\right\}\right) \neq \emptyset$. Hence we have already chosen $\left.x(\mathcal{W}) \in H_{\alpha}+\right\} \cap\left(H \backslash \bigcup\left\{c l_{\theta}(\overline{U(x)}): x \in\right.\right.$ $\left.\left.H^{\prime}\right\}\right) \subseteq H \cap(X \backslash H)$ a contradiction. Hence $X=H^{*}$ and $|X| \leq k 2^{\tau}$.

Now we use $U W(X), \psi w_{\theta}(X)$ and $h \theta-a L(X)$ to present a variation of the Theorem 2.23 in [6]. The proof of Theorem 3.2 follows step by step the proof of Theorem 2.23 in [6].

Theorem 3.2. If $X$ is a $T_{1}$ space then $|X| \leq U W(X) \psi w_{\theta}(X)^{h \theta-a L(X)}$.

Proof. Let $U W(X) \leq k, h \theta-a L(X) \leq \tau$ and $\psi w_{\theta}(X) \leq \lambda$. For all $x \in X$, let $\mathcal{U}_{x}$ be a family of open neighborhood of $x$ such that $\left|\mathcal{U}_{x}\right| \leq \lambda$ and $U w(x)=$ $\bigcap\left\{\operatorname{cl}_{\theta}(\bar{U}): \quad U \in \mathcal{U}_{x}\right\}$. By trasfinite induction we construct two families $\left\{H_{\alpha}: \alpha \in \tau^{+}\right\}$and $\left\{\mathcal{B}_{\alpha}: \alpha \in \tau^{+}\right\}$such that:

1. $\left\{H_{\alpha}: \alpha \in \tau^{+}\right\}$is an increasing sequence of subsets of $X$;

2. $\left|H_{\alpha}\right| \leq k \lambda^{\tau}$ for all $\alpha \in \tau^{+}$;

3. if $\left\{H_{\beta}: \beta \in \alpha\right\}$ are defined for some $\alpha \in \tau^{+}$, then $\mathcal{B}_{\alpha}=\bigcup\left\{\mathcal{U}_{x}: x \in\right.$ $\left.\bigcup\left\{U w(y): y \in \bigcup\left\{H_{\beta}: \beta \in \alpha\right\}\right\}\right\}$;

4. if $\alpha \in \tau^{+}$and $\mathcal{W} \in\left[\mathcal{B}_{\alpha}\right]^{\leq \tau}$ is such that $X \backslash\left(\bigcup\left\{c l_{\theta}(\bar{U}): U \in \mathcal{W}\right\}\right) \neq \emptyset$ then $H_{\alpha}-\left(\bigcup\left\{c l_{\theta}(\bar{U}): U \in \mathcal{W}\right\}\right) \neq \emptyset$. 
Let $\alpha \in \tau^{+}$and $\left\{H_{\beta}: \beta \in \alpha\right\}$ be already defined. For all $\mathcal{W}$ as in 4., choose a point $x(\mathcal{W}) \in X \backslash\left(\bigcup\left\{c l_{\theta}(\bar{U}): U \in \mathcal{W}\right\}\right)$ and let $C_{\alpha}$ be the set of these points.

Let $H_{\alpha}=\bigcup\left\{H_{\beta}: \beta \in \alpha\right\} \cup C_{\alpha}$. Then $\mid H_{\alpha} \leq k \lambda^{\tau}$.

Let $H=\bigcup\left\{H_{\alpha}: \quad \alpha \in \tau^{+}\right\}$and $H^{*}=\bigcup\{U w(x): x \in H\} \supseteq H$. Then $\left|H^{*}\right| \leq k \lambda^{\tau}$.

We want to prove that $X=H^{*}$. Suppose that there exists a point $q \in X \backslash H^{*}$. Then $q \notin U w(x), \forall x \in H$. Hence for all $x \in H$ there is $U(x) \in \mathcal{U}_{x}$ such that $q \notin c l_{\theta}(\overline{U(x)})$. From $h \theta-a L(X) \leq \tau$ choose $H^{\prime} \in[H] \leq \tau$ such that $H \subseteq \bigcup\left\{c l_{\theta}(\overline{U(x)}): x \in H^{\prime}\right\}$. Let $\mathcal{W}=\left\{\overline{U(x)}: x \in H^{\prime}\right\}$. We have that $H^{\prime} \subseteq$ $H_{\alpha}$ for some $\alpha \in \tau^{+}$and $\mathcal{W} \in\left[\mathcal{B}_{\alpha+1}\right] \leq \tau$ and $X \backslash\left(\bigcup\left\{c l_{\theta}(\bar{U}): U \in \mathcal{W}\right\}\right) \neq \emptyset$. Hence we have already chosen $x(\mathcal{W}) \in X \backslash\left(\bigcup\left\{c l_{\theta}(\bar{U}): U \in \mathcal{W}\right\} \subseteq X \backslash H\right.$ and $x(\mathcal{W}) \in H$ a contradiction. Hence $X=H^{*}$ and $|X| \leq k \lambda^{\tau}$.

Corollary 3.1. If $X$ is a Urysohn space then $|X| \leq \psi_{\theta}(X)^{h \theta-a L(X)}$.

\section{The Urysohn point separating weight}

Definition 4.1. [5] A Hausdorff point separating open cover $\mathcal{S}$ for a space $X$ is an open cover of $X$ having the property that for each distinct points $x, y \in X$ there exists $S \in \mathcal{S}$ such that $x \in S$ and $y \notin \bar{S}$.

The Hausdorff point separating weight of a space $X$ is

$H \operatorname{psw}(X)=\min \{\tau: X$ has a Hausdorff point separating open cover $\mathcal{S}$ such that each point of $X$ is contained in at most $\tau$ elements of $\mathcal{S}$ \}.

Following the same idea as in [5] we introduce the following definition:

Definition 4.2. A Urysohn point separating open cover $\mathcal{S}$ for a space $X$ is an open cover of $X$ having the property that for each distinct points $x, y \in X$ there exists $S \in \mathcal{S}$ such that $x \in S$ and $y \notin c l_{\theta}(\bar{S})$.

Definition 4.3. The Urysohn point separating weight of a Urysohn space $X$ is the cardinal:

$\operatorname{Upsw}(X)=\min \{\tau: X$ has a Urysohn point separating open cover $\mathcal{S}$

such that each point of $\mathrm{X}$ is contained in at most $\tau$ elements of $\mathcal{S}\}+\aleph_{0}$.

Note that $H p s w(X) \leq U p s w(X)$, for every Urysohn space $X$.

The proof of the following theorem follows step by step the proof of Theorem 20 in [5].

Theorem 4.1. If $X$ is a Urysohn space then $n w(X) \leq U p s w(X)^{\theta-a L_{c}(X)}$. 
Proof. Let $\theta-a L_{c}(X)=k$ and $\mathcal{S}$ a Urysohn point separating open cover for $X$ such that for each $x \in X,\left|\mathcal{S}_{x}\right| \leq \lambda$, where $\mathcal{S}_{x}$ is the collection of members of $\mathcal{S}$ containing $x$.

We first show that $d(X) \leq \lambda^{k} . \forall \alpha<k$ construct a subset $D_{\alpha}$ of $X$ such that:

1. $\left|D_{\alpha}\right| \leq \lambda^{k}$

2. if $\mathcal{U}$ is a subcollection of $\bigcup\left\{\mathcal{S}_{x}: x \in \bigcup_{\beta<\alpha} D_{\beta}\right\}$ such that $|\mathcal{U}| \leq k$ and if $X \backslash \bigcup c l_{\theta}(\overline{\mathcal{U}}) \neq \emptyset$ we have that $D_{\alpha} \backslash \bigcup c l_{\theta}(\overline{\mathcal{U}}) \neq \emptyset$.

Such a $D_{\alpha}$ can be constructed since the member of possible $\mathcal{U}^{\prime} s$ at the $\alpha$ th stage of construction is $\leq\left(\lambda^{k} k \lambda\right)^{k}=\lambda^{k}$.

Let $D=\bigcup_{\alpha<k^{+}} D_{\alpha}$. We have that $|D| \leq \lambda^{k}$. We want to prove that $\bar{D}=X$. Suppose that there exists $p \in X \backslash \bar{D}$, since $U p s w(X) \leq \lambda, \forall x \in \bar{D}$, there exists $V_{x} \in \mathcal{S}_{x}: p \notin c l_{\theta}\left(\overline{V_{x}}\right)$. Since $x \in \bar{D}, V_{x} \cap D \neq \emptyset$. Let $y \in V_{x} \cap D$, so $V_{x} \in \bigcup\left\{\mathcal{S}_{y}: y \in D\right\}$. Put $\mathcal{W}=\left\{V_{x}: x \in \bar{D}\right\} \subseteq \bigcup\left\{\mathcal{S}_{y}: y \in D\right\}$. $\mathcal{W}$ is an open cover of $\bar{D}$ and since $\theta-a L_{c}(X) \leq k$, there exists $\mathcal{W}^{\prime} \subseteq \mathcal{W}$ with $\left|\mathcal{W}^{\prime}\right| \leq k$ such that $\bar{D} \subseteq \bigcup\left\{c l_{\theta}(\bar{V}): V \in \mathcal{W}^{\prime}\right\}$ and $p \notin \bigcup\left\{c l_{\theta}(\bar{V}): V \in \mathcal{W}^{\prime}\right\}$ and this contradicts 2 ..

Since $d(X) \leq \lambda^{k}$ we have that $|\mathcal{S}| \leq \lambda^{k}$.

Let $\mathcal{N}=\{X \backslash S: \quad S$ is the union of at most $k$ members of $\mathcal{S}\}$. $|\mathcal{N}| \leq \lambda^{k}$ and $\mathcal{N}$ is a network for $X$.

Theorem 4.2. If $X$ is a Urysohn space then $|X| \leq U p s w(X)^{\theta-a L_{c}(X) \psi(X)}$.

Proof. If $X$ is a $T_{1}$ space then $|X| \leq n w(X)^{\psi(X)}$ and using the theorem above we have that $|X| \leq n w(X)^{\psi(X)} \leq U p s w(X)^{\theta-a L_{c}(X) \psi(X)}$.

\section{Acknowledgement}

The authors are very grateful to J. Porter for suggesting Example 3.3 .

\section{References}

[1] M.Bell, J.Ginsburg, G.Woods, Cardinal inequalities for topological space involving the weak Lindelöf number, Pacific J. Math (1978).

[2] A. Bella, F. Cammaroto, On the cardinality of Urysohn spaces, Canad. Math. Bull. Vol. 31 (2) (1988). 
[3] M.Bonanzinga, On the Hausdorff number of a topoloical space, Houston J. Math., 39(3), 1013-1030 (2013).

[4] M. Bonanzinga, F. Cammaroto, M.V. Matveev, On the Urysohn number of a topological space, Quaest. Math. 34, 441-446 (2011).

[5] M. Bonanzinga, N. Carlson, M.V. Cuzzupé, D. Stavrova, More on the cardinality of a topological space, preprint (2017).

[6] M. Bonanzinga, D. Stavrova, P. Staynova, Separation and CardinalitySome New Results and Old Questions, Topol. Appl. 221, 556-569 (2017).

[7] A. Charlesworth, On the cardinality of a topological space, Proc. of the Am. Math. Soc., 66, 1, 138-142 (1977).

[8] F. Cammaroto, Lj. Kočinac, On $\theta$-tightness, (1992).

[9] F. Cammaroto, A. Catalioto, J. Porter, Cardinal functions $F_{\theta}(X)$ and $t_{\theta}(X)$ for H-closed spaces, Quest. Math. 37, 309-320, (2014).

[10] N. Carlson, J. Porter, On the cardinality of Hausdorff spaces and $\mathrm{H}$ closed spaces, Topology Appl. (2017), DOI.

[11] J. de Groot, Discrete subspaces of Hausdorff spaces, Bull. Acad. Pol. Sci., Sr. Sci. Math. Astron. Phys. 13, 537-544 (1965).

[12] Ryszard Engelking, General Topology, Heldermann Verlag (1989).

[13] K.P. Hart, J. Nagata e J.E Vaughan, Encyclopedia of General Topology, Elsevier Science B.V. (2004).

[14] R.E. Hodel, Cardinal functions. I, Handbook of Set-Theoretic Topology, North Holland, 1-61 (1984).

[15] R.E. Hodel, Arhangelskiis solution to Alexandroff problem: A survey, Topol. Appl. 15321992217 (2006).

[16] I. Juhász, Cardinal functions in topology, Math. Centr. (1979).

[17] M.N. Mukherjee, A. Sengupta, S.K. Ghosh, On some cardinal functions concerning Katětov extensions of infinite discrete spaces (2009).

[18] B. Sapirovskii, Canonical sets and character, density and weight in compact spaces, Soviet. Math. Dokl. 15, 1282-1287 (1974). 
[19] S. Shu-Hao, Two new topological cardinal inequalities, Am. Math. Soc. 104, 313-316 (1988).

[20] D. Stavrova, Separation pseudocharacter and the cardinality of topological spaces, Top. Proc., 333-343 (2000).

[21] Lynn A. Steen, J. Arthur Seebach, Counterexamples in Topology, Holt, Rinehart and Winston, Inc (1970).

[22] J. Porter, General Topology Notes (2009).

[23] Jack R. Porter, R. Grant Woods, Extensions and Absolutes of Hausdorff Spaces, Springer-Verlag (1980).

[24] N.V. Veličko, H-closed topological spaces, Mat. Sb. 70, 98-112 (1966).

[25] S. Willard, U.N.B. Dissanayake, The almost Lindelöf degree, Canad. Math. Bull. Vol. 27 (4), 452-455 (1984). 